\title{
Surface morphology and property of UV-cured film containing photopolymerizable polysiloxane-based nanogels with initiating capability
}

\author{
Meng Wei ${ }^{1,2}$ · Jun-yi Han ${ }^{1,2} \cdot$ Yan-jing Gao ${ }^{2}$ Sheng-ling Jiang ${ }^{3} \cdot$ Fang Sun $^{1,2}$
}

Received: 23 December 2018 / Accepted: 8 August 2019 / Published online: 17 August 2019

(c) The Author(s) 2019

\begin{abstract}
We have synthesized a kind of photopolymerizable polysiloxane-based nanogel with intramolecular-initiating capability based on 4-hydroxybenzophenone (HBP), isobornyl methacrylate (IBMA), urethane dimethacrylate (UDMA) and 2-isocyanatoethyl methacrylate (IEM) and methacrylate-modified polysiloxane (PSMA). The nanogel possesses low migration of photolysis fragments and can dramatically reduce the volumetric shrinkage. In this work, the influences of the nanogels on the properties of UV-cured films prepared with nanogel and triethylene glycol dimethacrylate (TEGDMA) including rheology, glass transition temperature $\left(T_{\mathrm{g}}\right)$, tensile property, thermostability, surface morphology and surface energy of the UV-cured films were systematically studied. The results show that with the addition of nanogels at different loading levels, the viscosity increased fast and reached up to $9600 \mathrm{mPa}$ s. The increasing the content of nanogels in formulations resulted in the enhancement of thermal stability and elongation at break of the UV-cured films, and the formation of a more homogeneous network. Moreover, by adding the nanogels, the tensile strength of the cured films and surface energy declined. The enrichment of the nanogel on the surface can generate a more hydrophobic surface. Thus, the nanogels have potentially practical value in tuning structure and properties of polymer networks.
\end{abstract}

\section{Graphic abstract}

The polymerizable nanogels, which can initiate photopolymerization and reduce volumetric shrinkage, have the remarkable capability in decreasing surface energy, enhancing thermal stability and elongation at break, and homogenizing structure of polymer networks.

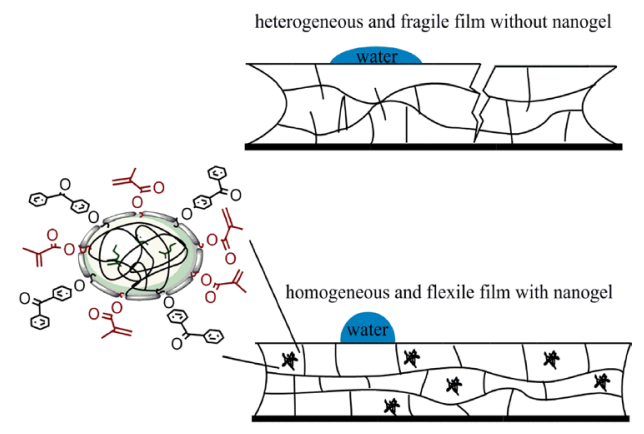

Keywords Nanogel $\cdot$ Photopolymerization $\cdot$ Mechanical property $\cdot$ Tenacity

Electronic supplementary material The online version of this article (https://doi.org/10.1007/s40090-019-00193-x) contains supplementary material, which is available to authorized users.

Fang Sun

sunfang60@yeah.net

Extended author information available on the last page of the article

\section{Introduction}

Photopolymer materials, which have a great number of advantages: a wide applied range, high sensitivity, selfprocessing nature and relatively low cost, can be used in 
biomaterials [1], coatings [2], adhesives [3], printing inks [4], dental materials [5], holographic data storage [6], diffractive and refractive optical elements [7], solar concentrators [8], holographic interferometry and holographic sensors [9].

However, the photopolymer materials usually suffer from high volumetric shrinkage. In the polymerization process, the volumetric shrinkage occurs during crosslinking of the polymeric system and depends on the chemical composition and polymerization reaction. Previously, it was reported that various strategies are applied to reduce the volumetric shrinkage, involving the use of nanoparticle-photopolymer composites [10], liquid crystalline monomers [11], radical/cationic hybrid initiating systems [12], thiol-ene cross-linked systems [13], and nanogels [14].

Nanogels, formed by physically or chemically crosslinked polymer networks with nanoscale size, have been widely researched in the fields of tissue engineering [15], photonic materials [16], drug delivery [17], dental materials [18], modifiers for coatings and polymer composites [19]. In previous research, we reported various reactive nanogels that have shown the capability to reduce the shrinkage stress without significant influences on the mechanical properties of materials [20]. Recently, we have synthesized a series of nanogels with intramolecular-initiating capability for free radical photopolymerization and researched the photochemical properties and photoinitiation mechanism of the nanogels possessing both chromophoric groups and hydrogen donating sites [21]. However, we do not know yet how the nanogels affect the mechanical properties of UV-cured films. It is very important to develop the application of the nanogels. Therefore, dispersing nanogels with different loading levels into dimethacrylate monomer and then various properties of the formulations, including rheology, glass transition temperature $\left(T_{\mathrm{g}}\right)$, tensile properties and thermostability were evaluated. More importantly, the surface property, morphology and elemental composition of the polymers containing the nanogels also were investigated.

\section{Experimental}

\section{Materials}

Dual-end terminated methacrylate-modified polysiloxane (PSMA, Mn $=380$ ) was donated by Shin-Etsu Chemical Co. Ltd (Shanghai, China). Isobornyl methacrylate (IBMA) and triethylene glycol dimethacrylate (TEGDMA) were donated by Eternal Chemical Co. Ltd (Zhuhai, China). Diethyl thioether, urethane dimethacrylate (UDMA) and 2-isocyanatoethyl methacrylate (IEM) were purchased from Heowns Business License Co. Ltd (Tianjin, China).
2-Mercaptoethanol (ME) was supplied by Fuchen Chemical Reagents Factory (Tianjin, China). Isophorone diisocyanate (IPDI) was obtained from Qingdao Xinyutian Chemical Co. (Qingdao, China). Benzophenone (BP) and 4-hydroxybenzophenone (HBP) were purchased from Sinopharm Group Chemical Reagent Co. (Beijing, China). Azobisisobutyronitrile (AIBN) was purchased from Xilong Chemical Co. Ltd (Shantou, China). Triethanolamine (TEOA) and dibutyltindilaurate (DBTDL) were supplied by Shanghai Chemical Reagents Co. (Shanghai, China). Triethylamine (TEA), ethanol, ethyl acetate, hexane, acetonitrile, phosphorus pentoxide $\left(\mathrm{P}_{2} \mathrm{O}_{5}\right)$ and dichloromethane (DCM) were purchased from Beijing Chemical Works (Beijing, China). Dichloromethane (DCM) was dried using $\mathrm{P}_{2} \mathrm{O}_{5}$. The nanogels which are designated as Si-HBP10, Si-HBP20, SiHBP30, Si-HBP40 and Si-HBP50, respectively, to represent the 10, 20, 30, 40 and $50 \mathrm{~mol} \%$ component of HBP in the terminal groups of the nanogel were synthesized by a known procedure [21]. (See Scheme S1).

\section{Characterization}

Transmission electron microscopy (TEM) images were obtained by JEOL JEM-2010 high-resolution transmission electron microscopes. The accelerating voltage was $20.0 \mathrm{kV}$. The average size and size distribution of the nanogel were measured by dynamic light scattering (DLS) using a Zetasizer NanoZS (ZEN 3600, Malvern, Germany). All measurements were carried out three times in $0.01 \mathrm{w} / \mathrm{v} \%$ dichloromethane solutions at $25^{\circ} \mathrm{C}$ using a $633 \mathrm{~nm}$ wavelength beam at $90^{\circ}$ scattering angle. Dichloromethane diluting each dispersion was ultrafiltered through a $0.20-\mu \mathrm{m}$ membrane to remove dust.

The tensile properties of UV-cured films were measured by using a material testing instrument (Instron-1211, USA) at $25{ }^{\circ} \mathrm{C}$. The rate of extension was $10 \mathrm{~mm} \mathrm{~min}^{-1}$.

The surface morphology of the samples was investigated using an S-4700 Hitachi scanning electron microscope (SEM) with an accelerating voltage of $10.0 \mathrm{kV}$ combined with energy dispersive X-ray spectroscopy (EDS) for the determination of the elemental composition. The surface elements of the polymer samples were characterized using XPS (PHI Quantera II, Japan).

Water contact angles on the surface of polymer films were tested on a contact angle goniometer (OCA20, Data Physics Co., Germany). Three replicates were conducted for each sample.

\section{Preparation procedure of UV-cured film}

The different nanogels were added to TEGDMA in mass ratios of $10-30 \mathrm{wt} \%$ in $10 \%$ increments. HBP $(0.5 \mathrm{wt} \%$, 
relative to resin) was added to TEGDMA without the nanogels as the control. Samples were mechanically agitated until the nanogel was fully dispersed to obtain optically clear monomeric solutions. The solution was cast in a Teflon mold $(20 \mathrm{~mm} \times 5 \mathrm{~mm} \times 0.3 \mathrm{~mm})$ to form a liquid film with a thickness of $0.3 \mathrm{~mm}$. A coverglass was then placed to cover the film to ensure anaerobic conditions. The deposited liquid film was then exposed to a high-pressure mercury lamp [incident light intensity $5 \mathrm{~mW} \mathrm{~cm}{ }^{-2}$, recorded by UV radiometer (Photoelectric Instrument Factory)] for 2 min to obtain the polymer films. The nanogelmodified formulations and final conversion are listed in Fig. S1 and Table S1.

\section{Rheology test}

Viscosity measurements of the nanogel-modified TEGDMA resins were performed using NDJ-79 viscometer (Shanghai Changji Instrument Co. Ltd, China). A defined volume of the control and nanogel-modified resins were tested at $15^{\circ} \mathrm{C}$ under the following conditions: $75 \mathrm{r} \mathrm{min}^{-1}$, and a run time of $30 \mathrm{~s}$. Three replicates were conducted for each sample.

\section{Calculation of gel fraction and swellability}

The swellability and gel fraction of a polymer network correspond to its network density. By swelling polymer films of the tested networks, the swellability $(S)$ and gel fraction $(G)$ were determined (Eqs. 1, 2):

$S=\frac{m_{\text {swollen }}}{m_{\text {dry }}} \times 100 \%$,

$G=\frac{m_{\text {dry }}}{m_{\text {start }}} \times 100 \%$.

The films were weighed at the beginning $\left(m_{\text {start }}\right)$ and then submerged in ethanol for 7 days. The swollen the polymer samples were weighed in the swollen state $\left(m_{\text {swollen }}\right)$ and then dried in a vacuum oven to receive the dry mass of the polymer samples $\left(m_{\mathrm{dry}}\right)$.

\section{Thermal analysis}

The dynamic thermal mechanical analysis (DMTA) was performed on DMTA-IV (Rheometric Scientific Co.). Measurements were collected from -50 to $200{ }^{\circ} \mathrm{C}$ with a heating rate of $5{ }^{\circ} \mathrm{C} \mathrm{min}{ }^{-1}$, at a frequency of $1 \mathrm{~Hz}$. Thermogravimetry analysis (TGA) was performed on STA-449C thermogravimetric analysis instrument (NETZSCH Instrument Co., Germany) under a nitrogen atmosphere with a heating rate of $20{ }^{\circ} \mathrm{C} \mathrm{min}^{-1}$ in the temperature range of $25-500{ }^{\circ} \mathrm{C}$.

\section{Results and discussion}

\section{Morphology of nanogels}

Figure 1 shows that all the nanogels appeared as microspheres and the particle size of the nanogels gradually increases with the increase of the benzophenone group content in the nanogels. Besides, the adhesion between the nanogels became more pronounced with the increase of the benzophenone group content in the nanogels. It is found from Fig. S2 and Table S2 that the average diameters of SiHBP10 Si-HBP50 from DLS measurement are $82.4 \mathrm{~nm}$, $98.5 \mathrm{~nm}, 105.6 \mathrm{~nm}, 111.2 \mathrm{~nm}$ and $121.5 \mathrm{~nm}$, respectively, and the size distribution of nanogels increases gradually, which is consistent with TEM image results. The results may be attributed to the increased molecular weight of the nanogel with the increase of benzophenone content [21]. Moreover, the average diameters of the nanogels tested by DLS are slightly greater compared with those tested by TEM due to the transition of the assemblies from the swollen (DLS) to dried state (TEM).

\section{Viscosity test}

Viscosity measurements for uncured TEGDMA samples with various contents of the nanogels are shown in Fig. 2. With the addition of nanogels at different loading levels, the viscosity increased fast. At $30 \mathrm{wt} \%$ nanogel loading, viscosity is quite high since the matrix phase is approaching a continuous interphase. Furthermore, the viscosity of Si-HBP10 at $30 \mathrm{wt} \%$ is $3300 \mathrm{mPa}$ s, while the viscosity for Si-HBP50 is increased to $9600 \mathrm{mPa}$ s. This result is related to the increase of the size, volume fraction and molecular weight of the nanogels because the friction force and interfacial regions between the molecules may increase with the increase of the

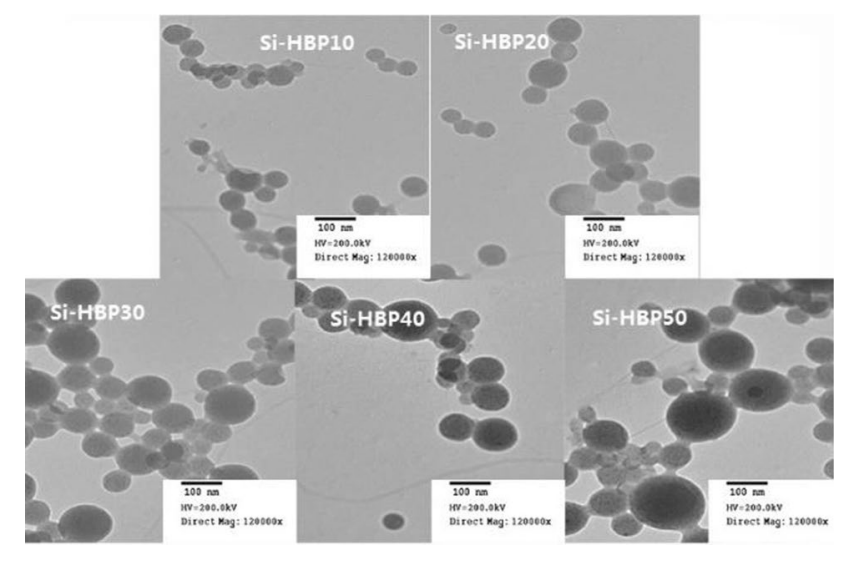

Fig. 1 TEM images of five kinds of the nanogels Si-HBP 

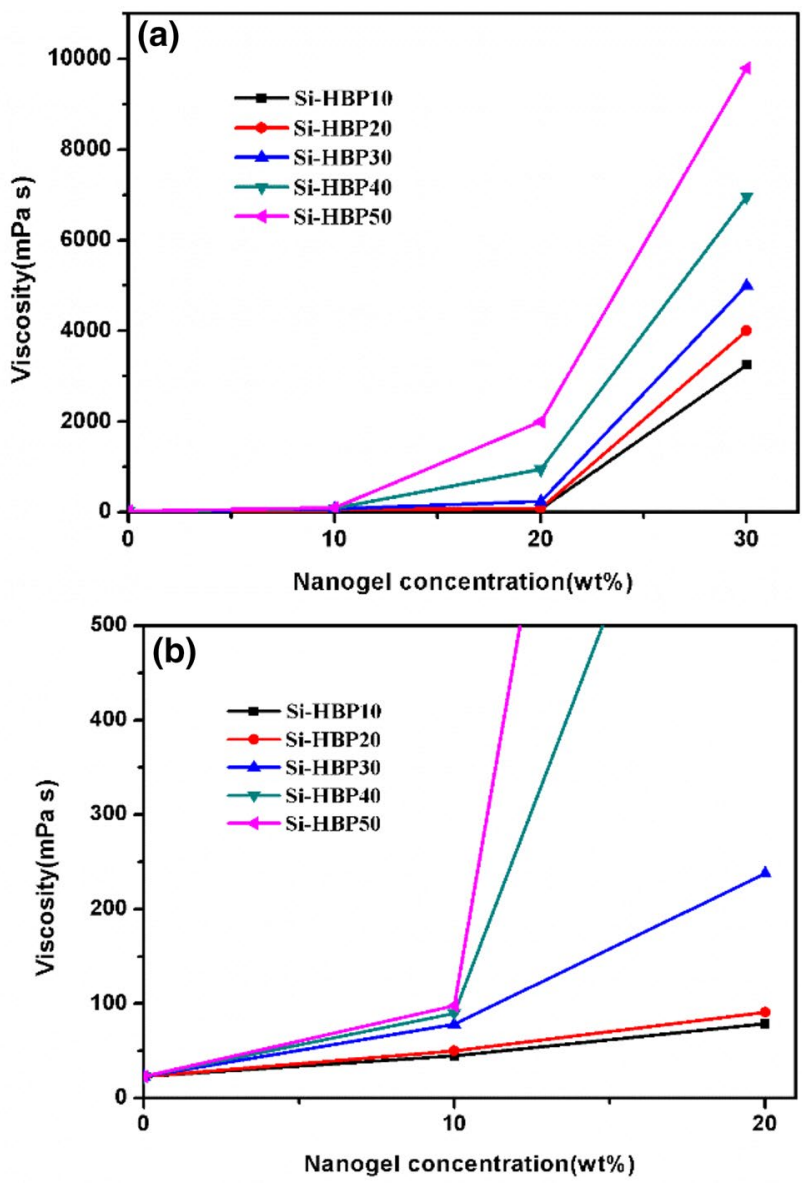

Fig. 2 Viscosity data for uncured TEGDMA systems added Si-HBP at various loading levels (a) and enlarged data (b)

size and molecular weight of nanogels, thereby leading to a high viscosity [16].

\section{Tensile properties of cured films}

The tensile properties of UV-cured films containing the nanogels were investigated as a vital indicator for the practical applications of UV-curable resins. From Table 1, it was found that the elongation at break of the cured films containing the nanogels is greater than that of the control system, whereas the tensile strength of the cured films containing the nanogels is lower than that of the control system. This is because the TEGDMA system without the nanogels has high double bond content, resulting in a high cross-linking degree. Moreover, the tensile strength of the cured films is improved with the increase in the benzophenone content of the nanogel, and the elongation at break exhibited a contrary trend. However, for the same nanogel, when the nanogel content increased, the elongation at break and the tensile strength remain virtually unchanged. This result is probably because the nanogels contain not only polysiloxane chain
Table 1 Tensile properties of cured films containing the nanogels

\begin{tabular}{lll}
\hline UV-cured films & $\begin{array}{l}\text { Tensile strength } \\
\text { (MPa) }\end{array}$ & $\begin{array}{l}\text { Elongation } \\
\text { at break (\%) }\end{array}$ \\
\hline TEGDMA & $45.5 \pm 1,6$ & $2.1 \pm 0.2$ \\
Si-HBP10 (10 wt\%) & $29.5 \pm 0.9$ & $8.6 \pm 0.8$ \\
Si-HBP20 (10 wt\%) & $32.5 \pm 1.1$ & $8.2 \pm 0.7$ \\
Si-HBP30 (10 wt\%) & $33.0 \pm 1.2$ & $7.8 \pm 0.6$ \\
Si-HBP40 (10 wt\%) & $34.8 \pm 1.3$ & $7.5 \pm 0.5$ \\
Si-HBP50 (10 wt\%) & $35.2 \pm 1.1$ & $7.4 \pm 0.5$ \\
Si-HBP10 (20 wt\%) & $30.4 \pm 1.5$ & $8.7 \pm 0.6$ \\
Si-HBP20 (20 wt\%) & $32.2 \pm 1.3$ & $8.3 \pm 0.7$ \\
Si-HBP30 (20 wt\%) & $32.8 \pm 0.9$ & $8.0 \pm 0.5$ \\
Si-HBP40 (20 wt\%) & $34.1 \pm 1.1$ & $7.9 \pm 0.7$ \\
Si-HBP50 (20 wt\%) & $34.2 \pm 1.2$ & $7.8 \pm 0.6$ \\
Si-HBP10 (30 wt\%) & $30.1 \pm 0.9$ & $8.9 \pm 1.0$ \\
Si-HBP20 (30 wt\%) & $32.0 \pm 1.4$ & $8.7 \pm 0.5$ \\
Si-HBP30 (30 wt\%) & $32.7 \pm 1.3$ & $8.1 \pm 0.4$ \\
Si-HBP40 (30 wt\%) & $34.0 \pm 1.2$ & $8.0 \pm 0.7$ \\
Si-HBP50 (30 wt\%) & $34.1 \pm 1.3$ & $7.9 \pm 0.5$ \\
\hline
\end{tabular}

segments with excellent flexibility, but also double bonds that can enhance the cross-linking degree of cured films.

\section{Dynamic mechanical thermal analysis of cured films}

The glass transition temperature $\left(T_{\mathrm{g}}\right)$ of a polymer network is an elective indicator in determining the performance and potential implementation of the polymer network. The samples of UV-cured films were investigated by DMTA to provide a direct link between chemical composition and mechanical behavior of the materials [22]. The $\tan \delta$ curve is sensitive to structural changes that occur at the molecular level during aging. A maximum in the $\tan \delta$ curve is associated with the movement of small groups and chains of molecules within the polymer structure, all of which are initially frozen in the polymer network [23]. It was proposed that by adding nanogels to a dimethacrylate formulation, the polymer network architecture can be altered, leading to more defined and tunable thermal transitions. The thermal transitions of the UV-cured films containing the nanogels were characterized by dynamic mechanical thermal analysis (DMTA). Only one damping peak is found for each $\tan \delta$ curve in Fig. 3, which suggests that the nanogels have good compatibility with acrylic monomers. Meanwhile, $T_{\mathrm{g}}$ of the polymer networks with nanogels is lower than that of the control, while intensity of damping peaks of polymer networks with nanogels is higher than that of the control The results demonstrate that the polymer networks have a low cross-linking density compared with the control, and the effective consolidation of the bulk TEGDMA and the nanogel phases is achieved. Furthermore, the Tan $\delta$ peak width of 

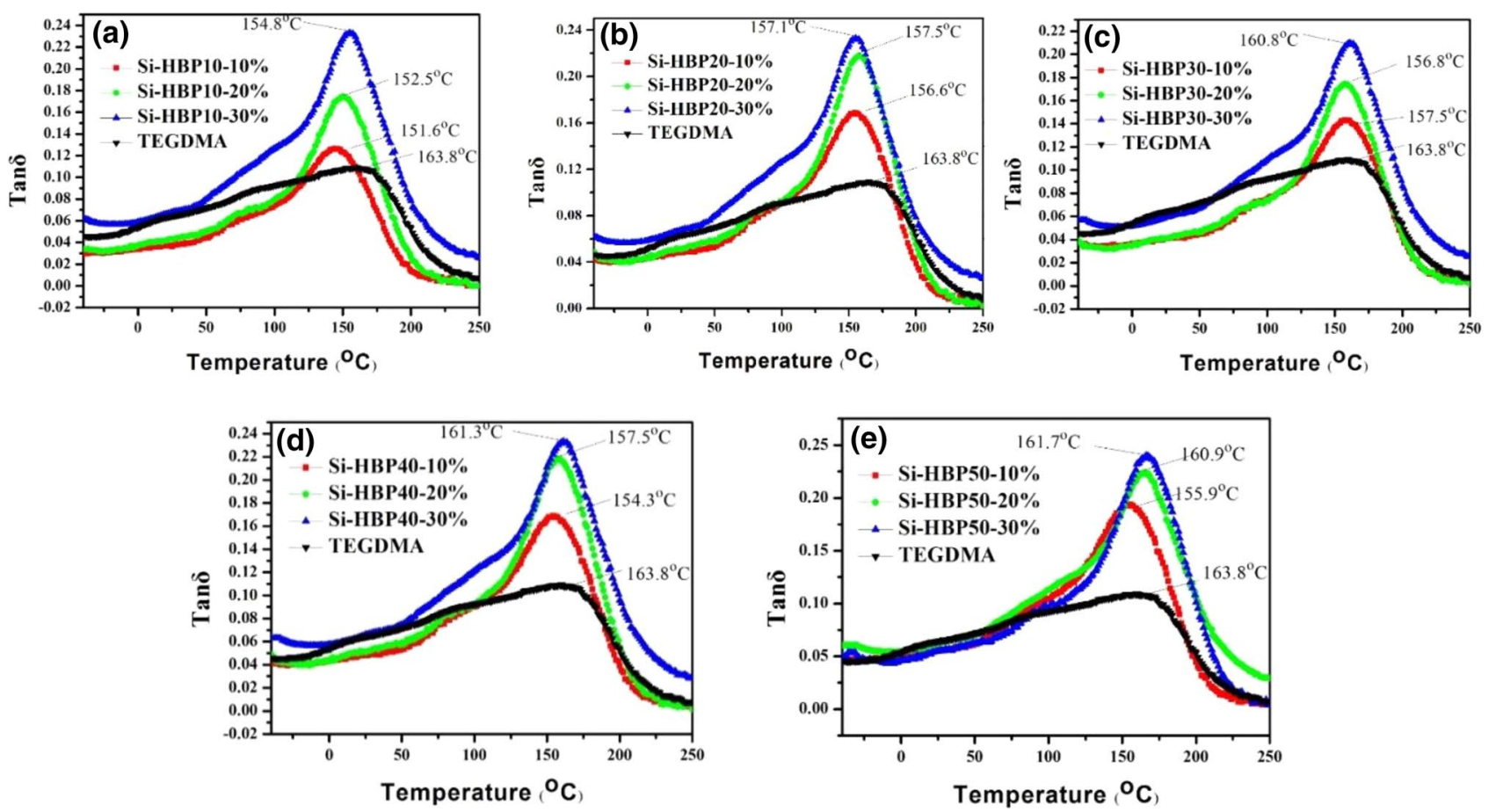

Fig. 3 Temperature spectra of Tan $\delta$ of cured films with the nanogel Si-HBP

the polymer film became narrower with the increase of addition amount of the nanogel, indicating that the cross-linked network of the polymer film became more homogeneous, and the nanogel can regulate the polymer network structure to a certain extent [16].

\section{Swellability and network density of cured films}

We have tested the swellability $(S)$ and gel fraction $(G)$ of nanogel/TEGDMA networks by swelling their polymer films (three films per network). The swellability and gel fraction of nanogel/TEGDMA networks in combination with their $T_{\mathrm{g}}$ can give the information about the crosslinking density of the polymer networks and help to understand the concept of nanogel/TEGDMA networks. The TEGDMA network as the control and the networks with the nanogels were analyzed. Except for both systems of SiHBP40 (30 wt\%) and Si-HBP50 (30 wt \%), the gel fraction (91.9-95.4\%) of the networks with nanogels were slightly less than that of the control network, while their swellability obviously higher than that of the control (Table 2). The reason was the migration of unreacted resin and the less cross-linked network with the lowest $T_{\mathrm{g}}$ [24]. It is still found from Table 2 and Fig. 3 that UV-cured films of Si-HBP40 (30 wt\%) system and Si-HBP50 (30 wt\%) system have the higher gel fraction $(\geq 96 \%)$ and more homogeneous network density compared to UV-cured film of TEGDMA without the nanogels, which indicates that the
Table 2 Gel fraction and swellability of polymer networks correlated with Tg

\begin{tabular}{llcc}
\hline UV-cured films & $\begin{array}{l}\text { Gel fraction } \\
(\mathrm{wt} \%)\end{array}$ & $\begin{array}{l}\text { Swellability } \\
(\mathrm{wt} \%)\end{array}$ & $T_{\mathrm{g}}\left({ }^{\circ} \mathrm{C}\right)$ \\
\hline TEGDMA & 96.0 & 5.2 & 163.8 \\
Si-HBP10 (10 wt\%) & 91.9 & 19.1 & 151.6 \\
Si-HBP20 (10 wt\%) & 93.5 & 11.5 & 156.6 \\
Si-HBP30 (10 wt\%) & 94.4 & 9.9 & 157.5 \\
Si-HBP40 (10 wt\%) & 95.0 & 9.2 & 154.3 \\
Si-HBP50 (10 wt\%) & 95.2 & 9.1 & 155.9 \\
Si-HBP10 (20 wt\%) & 92.4 & 15.3 & 152.5 \\
Si-HBP20 (20 wt\%) & 93.9 & 11.1 & 157.1 \\
Si-HBP30 (20 wt\%) & 94.9 & 9.6 & 156.8 \\
Si-HBP40 (20 wt\%) & 95.4 & 9.1 & 157.5 \\
Si-HBP50 (20 wt\%) & 95.9 & 7.2 & 160.9 \\
Si-HBP10 (30 wt\%) & 93.3 & 12.2 & 154.8 \\
Si-HBP20 (30 wt\%) & 94.5 & 9.8 & 157.5 \\
Si-HBP30 (30 wt\%) & 95.4 & 8.9 & 160.8 \\
Si-HBP40 (30 wt\%) & 96.0 & 8.8 & 161.3 \\
Si-HBP50 (30 wt\%) & 96.5 & 5.1 & 161.7 \\
\hline
\end{tabular}

architecture of the polymer network is altered by the addition of the nanogels. 
Fig. 4 TG and DTG curves of cured films with different contents of nanogels
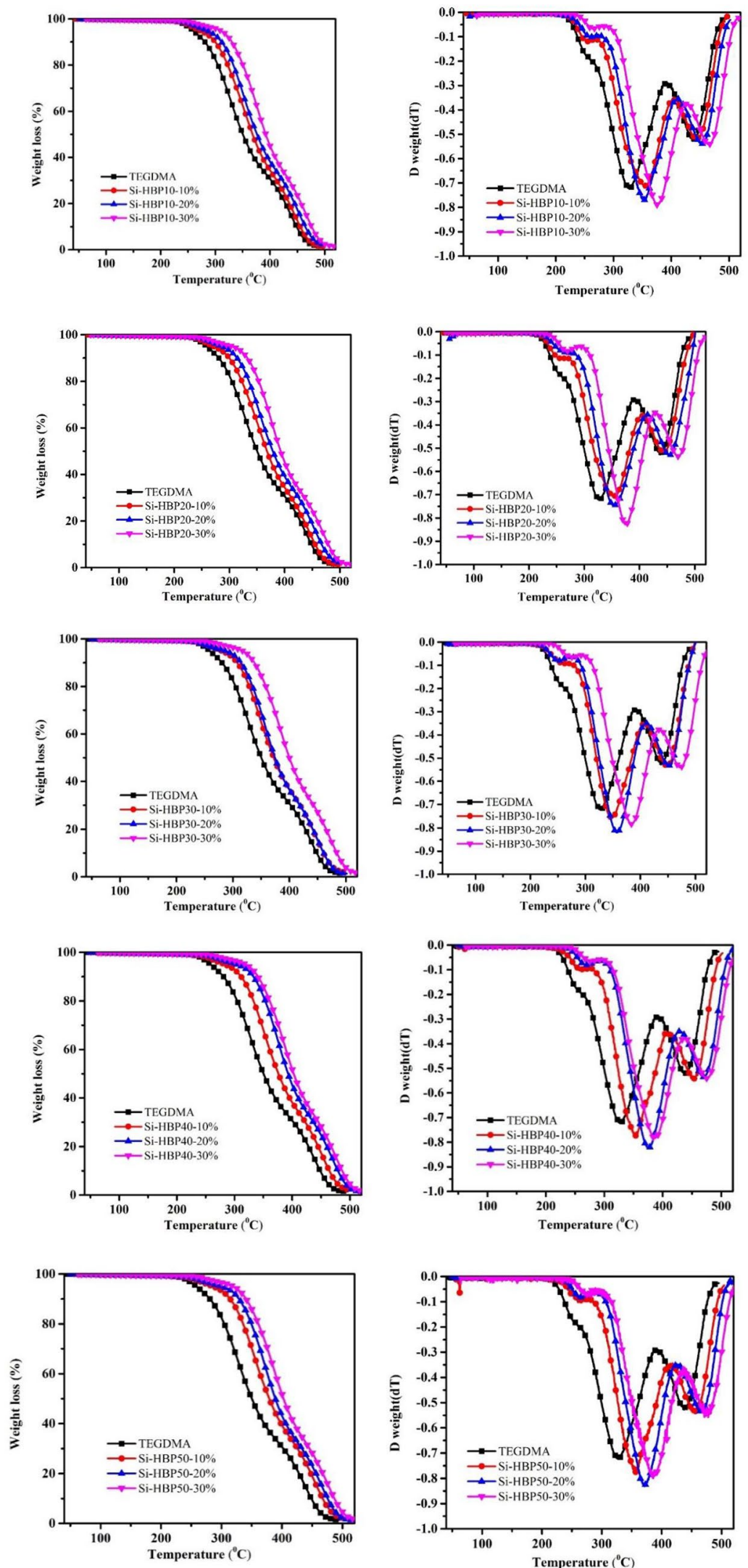
Table 3 Heat-resistant data of cured films with different contents of nanogels

\begin{tabular}{llll}
\hline UV-cured films & $T_{5 \%}\left({ }^{\circ} \mathrm{C}\right)$ & $T_{\max 1}\left({ }^{\circ} \mathrm{C}\right)$ & $T_{\max 2}\left({ }^{\circ} \mathrm{C}\right)$ \\
\hline TEGDMA & 256 & 328 & 438 \\
Si-HBP10-10\% & 282 & 355 & 441 \\
Si-HBP10-20\% & 290 & 352 & 452 \\
Si-HBP10-30\% & 302 & 378 & 464 \\
Si-HBP20-10\% & 280 & 352 & 441 \\
Si-HBP20-20\% & 294 & 353 & 454 \\
Si-HBP20-30\% & 304 & 378 & 468 \\
Si-HBP30-10\% & 283 & 349 & 445 \\
Si-HBP30-20\% & 296 & 359 & 452 \\
Si-HBP30-30\% & 310 & 384 & 472 \\
Si-HBP40-10\% & 281 & 354 & 452 \\
Si-HBP40-20\% & 304 & 377 & 465 \\
Si-HBP40-30\% & 319 & 387 & 474 \\
Si-HBP50-10\% & 287 & 355 & 456 \\
Si-HBP50-20\% & 301 & 371 & 465 \\
Si-HBP50-30\% & 316 & 391 & 471 \\
\hline
\end{tabular}

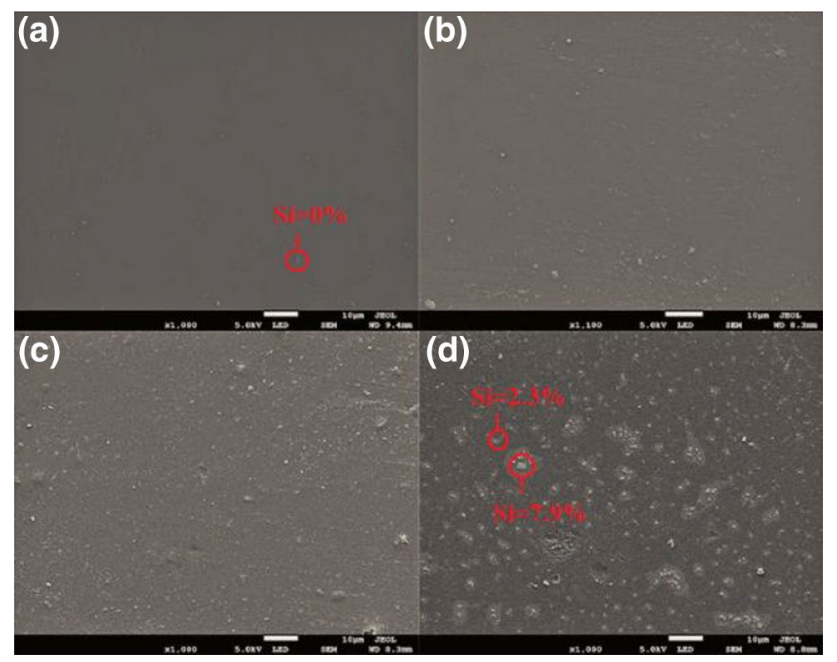

Fig. 5 SEM images of polymer films containing Si-HBP50 a TEGDMA, b $10 \%$ mass fraction, c $20 \%$ mass fraction, d $30 \%$ mass fraction

\section{Thermogravimetric analysis of cured films}

The thermal stability of polymer network is a very important property. The thermal stability of the UV-cured films containing the nanogels was investigated by thermogravimetric analysis under nitrogen atmosphere. Figure 4 shows the TG and DTG curves of the UV-cured films, and detailed parameter values obtained from the TG and DTG curves are listed in Table 3. The thermal degradation processes of the UV-cured films underwent single-step degradations with three DTG

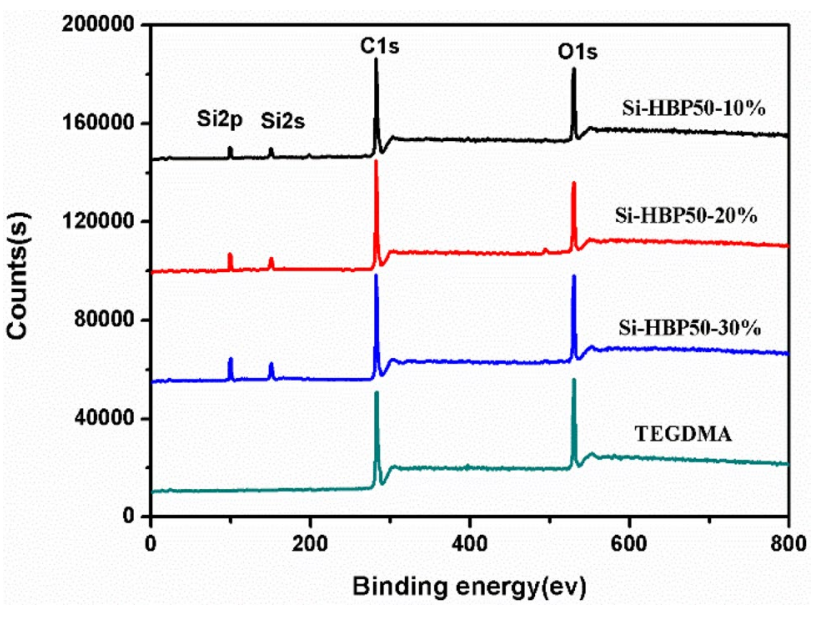

Fig. 6 XPS survey spectra of polymer films containing different contents of Si-HBP50

peaks. The temperature at the first DTG peak was close to $T_{5 \%}$, which was attributed to the degradation of small molecules with low molecular weight. It can be easily found that $T_{\max 1}$ and $T_{\max 2}$ of the UV-cured films containing nanogels were higher than those of the TEGDMA control, indicating the addition of nanogels can improve the thermal stability of UV-cured films. Furthermore, the thermal stability of the UVcured films enhanced with the increase of the nanogel content. The results were related to relatively high mass fraction of polysiloxane containing stronger $\mathrm{Si}-\mathrm{O}$ bonds in the nanogel and high cross-linking degree.

\section{Surface morphology and elemental composition of cured films}

The surface morphologies of the polymer films containing different concentrations of Si-HBP50 were observed by SEM in Fig. 5. It is obvious that the surfaces of the films with the nanogels presented irregular protuberances that were not observed on the surface of the film of TEGDMA control. Further research on these protuberances by EDS shows that the content of silicon in these regions was higher than that in other areas. It is therefore conjectured that the enrichment of the polysiloxane-modified nanogel resulted in the formation of the irregular protuberances. Furthermore, the number of irregular protuberances was generally increased and they began to cluster as the content of the nanogel increased.

The abundance of $\mathrm{C}, \mathrm{O}$ and $\mathrm{Si}$ on the surface of polymer films containing Si-HBP50 was detected by XPS, as shown in Fig. 6. The content of Si can reflect the content of the nanogels because silicon only exists in the nanogel. Table S3 shows that when the addition amount of Si-HBP50 in polymer film was $10 \%, 20 \%$ and $30 \%$, the content of Si on the surface of 
Table 4 Contact angle and dispersion surface energy of cured films

\begin{tabular}{llc}
\hline UV-cured films & $\theta\left(\mathrm{H}_{2} \mathrm{O}\right) /^{\circ}$ & $\gamma_{S}^{d}\left(\mathrm{H}_{2} \mathrm{O}\right) /(\mathrm{mN} / \mathrm{m})$ \\
\hline TEGDMA & 43.5 & 164.5 \\
Si-HBP10 (10 wt\%) & 49.6 & 145.8 \\
Si-HBP20 (10 wt\%) & 64.9 & 112.1 \\
Si-HBP30 (10 wt\%) & 70.1 & 101.7 \\
Si-HBP40 (10 wt\%) & 55.4 & 136.0 \\
Si-HBP50 (10 wt\%) & 65.4 & 104.4 \\
Si-HBP10 (20 wt\%) & 73.2 & 80.2 \\
Si-HBP20 (20 wt\%) & 61.7 & 120.7 \\
Si-HBP30 (20 wt\%) & 71.6 & 98.2 \\
Si-HBP40 (20 wt\%) & 80.4 & 63.7 \\
Si-HBP50 (20 wt\%) & 59.4 & 116.9 \\
Si-HBP10 (30 wt\%) & 82.3 & 69.9 \\
Si-HBP20 (30 wt\%) & 69.4 & 97.4 \\
Si-HBP30 (30 wt\%) & 81.4 & 60.7 \\
Si-HBP40 (30 wt\%) & 71.6 & 98.2 \\
Si-HBP50 (30 wt\%) & 74.1 & 89.7 \\
\hline
\end{tabular}

the polymer film was $3.37 \%, 6.75 \%$, and $8.27 \%$, respectively. This is attributed the fact that the content of the polysiloxane component in the polymer film increased with the increase of the content of the nanogel. The increase trend of the content of $\mathrm{Si}$ is consistent with SEM images results.

\section{The dispersion surface energy and contact angle of UV-cured films}

According to Fowkes' study, the interface tension could be calculated by the following formulation:

$\gamma_{\mathrm{SL}}=\gamma_{\mathrm{SV}}-\gamma_{\mathrm{LV}} \cos \theta$

$\gamma_{\mathrm{SL}}=\gamma_{\mathrm{S}}+\gamma_{\mathrm{LV}}-2\left(\gamma_{L}^{d} \gamma_{S}^{d}\right)^{1 / 2}$

Here, $\gamma_{S}^{d}$ is the dispersion surface energy, $\gamma_{\mathrm{SL}}$ is the surface tension at the solid-liquid interface, $\gamma_{\mathrm{SV}}$ is at the solid-vapor interface and $\gamma_{\mathrm{LV}}$ is at the liquid-vapor interface.

$\mathrm{H}_{2} \mathrm{O}\left(\gamma_{\mathrm{LV}}=72.7 \mathrm{mN} \mathrm{m}^{-1}, \gamma_{L}^{d}=23.9 \mathrm{mN} \mathrm{m}^{-1}\right)$ was chosen to be the testing liquid. The contact angle $(\theta)$ of the testing liquid on the planar surface of solid polymeric films tested here. In according to $\theta$, the $\gamma_{S}^{d}$ values can be calculated via Eq. (3) and Eq. (4). $\theta$ and values of $\gamma_{S}^{d}$ are given in Table 4.

The data in Table 4 show that all systems containing the nanogels have larger contact angles $(\theta)$ compared to the control because of the enrichment of polysiloxane on the surface, which is consistent with the SEM results. Therefore, the enrichment of the nanogel on the surface can reduce the surface energy of polymer film and generate a more hydrophobic surface. However, there is no clear trend in the enlargement of contact angles $(\theta)$ for $\mathrm{H}_{2} \mathrm{O}$ and the reduction of dispersion surface energy $\left(\gamma_{S}^{d}\right)$ of the cured films based on nanogels content and the benzophenone concentration of the nanogels.

\section{Conclusion}

In this paper, we studied the properties of UV-cured films containing five kinds of photopolymerizable nanogels with the capability of initiating photopolymerization. The nanogels possessed an excellent compatibility with dimethacrylate. An increasing content of nanogels in the formulations resulted in the formation of more homogeneous networks, whose $\tan \delta$ peak became sharper. Swellability tests have elucidated the relationship between gel fraction, swellability and network density. The networks with nanogels have lower network density compared to the TEGDMA networks, but still present a significantly high gel fraction. Polymer networks containing the nanogels show better thermal stability than the TEGDMA networks, and the thermal stability depends on the nanogel loading and the benzophenone content within the nanogels. The enrichment of the nanogel on the surface can reduce the dispersion surface energy of UV-cured films and enhance the hydrophobicity of the cured film surface. The nanogels have potentially practical value in tuning structure and properties of polymer networks.

Acknowledgements The financial support from the National Key R\&D Program of China (2017YFB0307800), the Special Funds of Jiangsu Province Key Research and Development (BE2015058), and the National Natural Science Foundation of China (Grant no. 51273014), are gratefully acknowledged.

Open Access This article is distributed under the terms of the Creative Commons Attribution 4.0 International License (http://creativeco mmons.org/licenses/by/4.0/), which permits unrestricted use, distribution, and reproduction in any medium, provided you give appropriate credit to the original author(s) and the source, provide a link to the Creative Commons license, and indicate if changes were made.

\section{References}

1. Li S, Tuft B, Xu L, Polacco M, Clarke J, Guymon C, Hansen M (2015) Microtopographical features generated by photopolymerization recruit RhoA/ROCK through TRPV1 to direct cell and neurite growth. Biomaterials 53:95-106

2. Li C, Tan J, Gu J, Qiao L, Zhang B, Zhang Q (2016) Rapid and efficient synthesis of isocyanate microcapsules via thiol-ene photopolymerization in Pickering emulsion and its application in selfhealing coating. Compos Sci Technol 123:250-258

3. Besse V, Derbanne M, Pham T, Cook W, Pluart L (2016) Photopolymerization study and adhesive properties of self-etch adhesives containing bis (acyl) phosphine oxide initiator. Dent Mater 32:561-569 
4. Aphinyan S, Geethalakshmi K, Yeo J, Shakouri A, Ng T (2017) Numerical characterization of ultraviolet ink fluid agglomeration and the surfactant effect in nanoinkjet printing. Polym Adv Technol 28:1057-1064

5. Armstrong S, Breschi L, Özcan M, Pfefferkorn F, Ferrari M, Meerbeek B (2017) Academy of Dental Materials guidance on in vitro testing of dental composite bonding effectiveness to dentin/enamel using micro-tensile bond strength $(\mu \mathrm{TBS})$ approach. Dent Mater 33:133-143

6. Pramitha V, Das B, Joseph J, Joseph R, Sreekumar K, Kartha C (2016) High efficiency panchromatic photopolymer recording material for holographic data storage systems. Opt Mater $52: 212-218$

7. Malinauskas M, Farsari M, Piskarskas A, Juodkazis S (2013) Ultrafast laser nanostructuring of photopolymers: a decade of advances. Phys Rep 533:1-31

8. Akbari H, Naydenova I, Ahmed H, McCormack S, Martin S (2017) Development and testing of low spatial frequency holographic concentrator elements for collection of solar energy. Sol Energy 155:103-109

9. Liu H, Yu D, Mao D, Geng Y, Wang W (2016) Modeling swelling and absorption dynamics for holographic sensing in analytes sensitive photopolymer. Opt- Commun 368:95-104

10. Bayramoğlu G, Şeker M, Mudu M (2016) Preparation of methacrylated polyglycidol-POSS based silver nanoparticle containing nanocomposites via photopolymerization. Prog Org Coat 101:510-521

11. Chen G, Ni M, Peng $\mathrm{H}$ et al (2017) Photoinitiation and inhibition under monochromatic green light for storage of colored 3D images in holographic polymer-dispersed liquid crystals. ACS Appl Mater Interfaces 9:1810-1819

12. Škola $O$, Jašúrek B, Veselý $D$, Němec $P$ (2016) Mechanical properties of polymer layers fabricated via hybrid free radical-cationic polymerization of acrylate, epoxide, and oxetane binders. Prog Org Coat 101:279-287

13. Ware T, Perry Z, Middleton C, Iacono S, White T (2015) Programmable liquid crystal elastomers prepared by thiol-ene photopolymerization. ACS Macro Lett 4:942-946

14. Chen C, Han J, Sun F (2017) Gradient polymer networks formed by photopolymerization with self-floating polysiloxane-containing nanogel. Polym Adv Technol 28:312-318
15. Jiang Y, Chen J, Deng C, Suuronen E, Zhong Z (2014) Click hydrogels, microgels and nanogels: emerging platforms for drug delivery and tissue engineering. Biomaterials 35:4969-4985

16. Liu J, Rad I, Sun F, Stansbury J (2014) Photo-reactive nanogels as a means to tune properties during polymer network formation. Polymer Chem 5:227-233

17. Chen X, Xiao C et al (2017) one-pot synthesis of ph and Ros dual responsive nanogels for anti-cancer drug delivery. J Control Release 259:e154

18. Liu J, Stansbury J (2014) RAFT-mediated control of nanogel structure and reactivity: chemical, physical and mechanical properties of monomer-dispersed nanogel compositions. Dent Mater 30:1252-1262

19. Lu H, Jin X, Barros M, Stansbury J (2015) Shrinkage and shrinkage stress control in highly-filled nanogel-modified composite. Dent Mater 31:e22-e23

20. Chen C, Liu J, Sun F, Stansbury J (2014) Control of microstructure and gradient property of polymer network by photopolymerizable silicone-containing nanogel. J Polym Sci Part A Polym Chem 52:2830-2840

21. Han J, Jiang S, Gao Y, Sun F (2016) Intramolecular-initiating photopolymerization behavior of nanogels with the capability of reducing shrinkage. J Mater Chem C 4:10675-10683

22. Candan Z, Gardner D, Shaler S (2016) Dynamic mechanical thermal analysis (DMTA) of cellulose nanofibril/nanoclay/pMDI nanocomposites. Compos B Eng 90:126-132

23. Grindy S, Learsch R, Mozhdehi D et al (2015) Control of hierarchical polymer mechanics with bioinspired metal-coordination dynamics. Nat Mater 14:1210

24. Gorsche C, Griesser M, Gescheidt G, Moszner N, Liska R (2014) $\beta$-Allyl sulfones as addition-fragmentation chain transfer reagents: a tool for adjusting thermal and mechanical properties of dimethacrylate networks. Macromolecules 47:7327-7336

Publisher's Note Springer Nature remains neutral with regard to jurisdictional claims in published maps and institutional affiliations.

\section{Affiliations}

\section{Meng Wei ${ }^{1,2}$ - Jun-yi Han ${ }^{1,2} \cdot$ Yan-jing Gao ${ }^{2}$. Sheng-ling Jiang ${ }^{3}$. Fang Sun ${ }^{1,2}$}

1 State Key Laboratory of Chemical Resource Engineering, Beijing University of Chemical Technology, Beijing 100029, People's Republic of China

2 College of Science, Beijing University of Chemical Technology, Beijing 100029, People's Republic of China
College of Mechanical and Electrical Engineering, Beijing University of Chemical Technology, Beijing 100029, People's Republic of China 